\title{
Numerical prediction of the effective coefficient of thermal expan- sion of 3D braided C/SiC composite
}

\author{
Yingjie $\mathrm{Xu}^{1, \mathrm{a}}$, Weihong Zhang ${ }^{1}$ \\ ${ }^{1}$ Key Laboratory of Contemporary Design \& Integrated Manufacturing Technology, Northwestern Polytechnical University, 710072, Xi'an, \\ Shaanxi, China
}

Received 20 May 2009, Accepted 30 September 2009

\begin{abstract}
This paper is focused on the microstructure modeling and evaluation of effective coefficient of thermal expansion (CTE) of 3D braided carbon fiber-reinforced silicon carbide composites (C/SiC). Regarding the multi-scale characteristics of the composite, the microstructure modeling is carried out sequentially from the fiber scale to tow scale. Effective elastic properties are obtained based on the sequential homogenization from the fiber scale to the tow scale. A stain energy model is developed for the prediction of the effective CTE of composite materials. This model is based on the relationship established between the strain energy of the microstructure and that of the homogenized equivalent model under specific thermo-elastic boundary conditions. Expressions of closed-form are derived for the effective CTE in terms of the strain energy and effective elastic tensor. Numerical results obtained by the proposed model show a good agreement with the results measured experimentally.
\end{abstract}

Key words: 3D braided C/SiC composite, Coefficient of thermal expansion, Microstructure modeling, Strain energy, Numerical prediction

\section{Introduction}

Under extreme high-temperature environment, the basic concern is to use materials which can maintain their superior character during exposure to hostile temperature environment ranging between $800^{\circ} \mathrm{C}$ and $1100^{\circ} \mathrm{C}[1] . \mathrm{C} / \mathrm{SiC}$ composites have been purposefully engineered according to these service requirements. $3 \mathrm{D}$ braided $\mathrm{C} / \mathrm{SiC}$ composites are widely used in high temperature air-breathing, space, and nuclear applications [1]. Introduction of $\mathrm{SiC}$ matrix into composites has boosted the possibility of having materials that could provide dimensional stability along with sustained thermo-physical properties in an environment prone to rapid corrosion and severe thermal shock.

Complicated spatial architecture and material heterogeneity of $3 \mathrm{D}$ braided $\mathrm{C} / \mathrm{SiC}$ composites constitute the challenges to understand their physical properties. Revelation of the intrinsic relations between the effective physical properties and the complex materials microstructure is thus the key for practical applications of $3 \mathrm{D}$ braided $\mathrm{C} / \mathrm{SiC}$ composites. The common numerical approach for characterizing the composite materials is to create a representative volume cell (RVC) that captures the major features of the underlying microstructure. A variety of RVC models and strategies were proposed to determine mechanical properties of 3D braided composite [2-12], but the work for characterization of $3 \mathrm{D} \mathrm{C} / \mathrm{SiC}$ composites has been limited compared to polymer and metal matrix composites.

This study aims at the microstructure modeling and numerical prediction of effective CTE of 3-D braided $\mathrm{C} / \mathrm{SiC}$ composite. Regarding the multi-scale characteristics of the composite, the microstructure modeling is carried out se-

\footnotetext{
${ }^{\text {a }}$ Corresponding author: xyj1014@gmail.com
}

quentially from the fiber-scale to tow-scale. Effective thermal-elastic properties are obtained based on the sequential homogenization. A stain energy model is developed for the prediction of the effective CTE. Numerical results obtained by the proposed model and are then compared with the results measured experimentally.

\section{Strain energy model for evaluation of CTE of composite}

In the present work, the relationship between the strain energy of unit cell and homogenized equivalent model is studied for 3D orthotropic materials. To make things clear, consider a unit cell model with heterogeneous microstructure depicted in Fig. 1a. It consists of two material phases (the dark color represent the fiber while the light color represent the matrix) and can be regarded as a homogenized equivalent model depicted in Fig. 1 b.

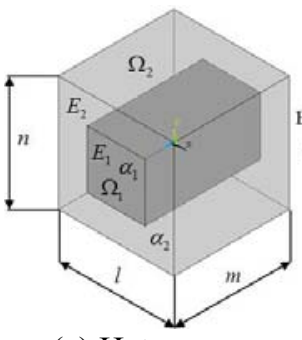

(a) Heterogeneous microstructure

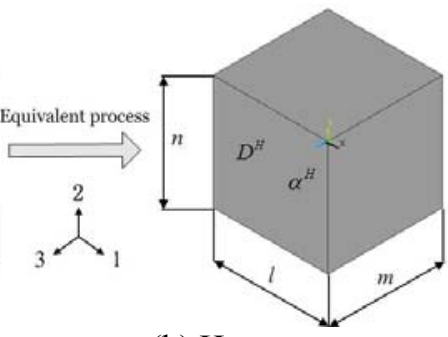

(b) Homogeneous equivalent model
Fig. 1. Illustration of RVC

In thermo-elastic regime, the macroscopic behaviors of the RVC can be characterized by the effective stress tensor $\bar{\sigma}$ and strain tensor $\bar{\varepsilon}$ over the homogeneous equivalent model: 


$$
\overline{\boldsymbol{\sigma}}=\boldsymbol{D}^{H}\left(\overline{\boldsymbol{\varepsilon}}-\boldsymbol{\alpha}^{H} \Delta T\right)
$$

where $\Delta T$ is the temperature increase. $\boldsymbol{D}^{H}$ and $\boldsymbol{\alpha}^{H}$ represent the effective stiffness matrix and thermal expansion coefficient matrix, respectively. Further more, $\boldsymbol{D}^{H}$ and $\boldsymbol{\alpha}^{H}$ can be written as:

$$
\begin{gathered}
\boldsymbol{D}^{H}=\left[\begin{array}{cccccc}
D_{1111}^{H} & D_{1122}^{H} & D_{1133}^{H} & 0 & 0 & 0 \\
D_{1122}^{H} & D_{2222}^{H} & D_{2233}^{H} & 0 & 0 & 0 \\
D_{1133}^{H} & D_{2233}^{H} & D_{3333}^{H} & 0 & 0 & 0 \\
0 & 0 & 0 & D_{1212}^{H} & 0 & 0 \\
0 & 0 & 0 & 0 & D_{2323}^{H} & 0 \\
0 & 0 & 0 & 0 & 0 & D_{3131}^{H}
\end{array}\right] \\
\alpha^{H}=\left[\begin{array}{lll}
\alpha_{11}^{H} & \alpha_{22}^{H} & \alpha_{33}^{H}
\end{array}\right]^{T}
\end{gathered}
$$

note that here $\boldsymbol{D}^{H}$ consists of known coefficients, which have been obtained by using the strain energy of microstructure but only considering elastic boundary conditions. Details about the implementation procedure were discussed in our previous work [14].

Thus, the relationship between effective stress and strain caused by the temperature increase of $\Delta T$ can be then given as:

$$
\begin{aligned}
\bar{\sigma}_{11}= & D_{1111}^{H}\left(\bar{\varepsilon}_{11}-\alpha_{11}^{H} \Delta T\right)+D_{1122}^{H}\left(\bar{\varepsilon}_{22}-\alpha_{22}^{H} \Delta T\right) \\
& +D_{1133}^{H}\left(\bar{\varepsilon}_{33}-\alpha_{33}^{H} \Delta T\right) \\
\bar{\sigma}_{22}= & D_{1122}^{H}\left(\bar{\varepsilon}_{11}-\alpha_{11}^{H} \Delta T\right)+D_{2222}^{H}\left(\bar{\varepsilon}_{22}-\alpha_{22}^{H} \Delta T\right) \\
& +D_{2233}^{H}\left(\bar{\varepsilon}_{33}-\alpha_{33}^{H} \Delta T\right) \\
\bar{\sigma}_{33}= & D_{1133}^{H}\left(\bar{\varepsilon}_{11}-\alpha_{11}^{H} \Delta T\right)+D_{2233}^{H}\left(\bar{\varepsilon}_{22}-\alpha_{22}^{H} \Delta T\right) \\
& +D_{3333}^{H}\left(\bar{\varepsilon}_{33}-\alpha_{33}^{H} \Delta T\right)
\end{aligned}
$$

The strain energy related to the RVC is equal to:

$$
C=\int_{\Omega} \sigma^{T} \varepsilon d \Omega
$$

note that $\sigma$ and $\varepsilon$ are the stress and strain tensor over the $\mathrm{RVC}$, respectively.

Now, consider the following three kinds of boundary condition depicted in Figs. 2, 3 and 4, respectively.

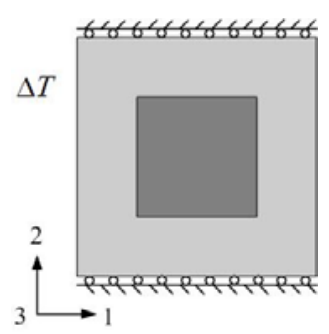

(a) 3-dirction cross-section

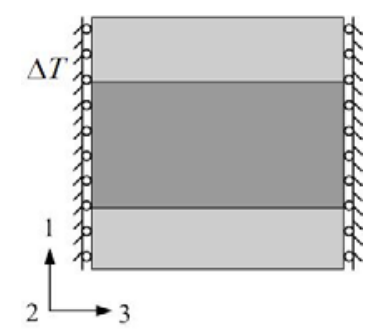

(b) 2-dirction cross-section

Fig. 2. RVC under boundary condition 1

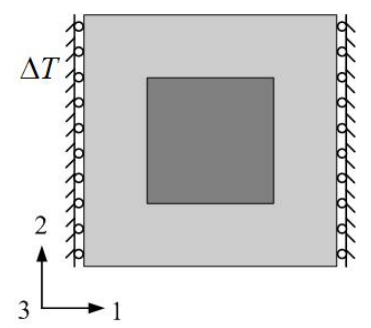

(a) 3-dirction cross-section

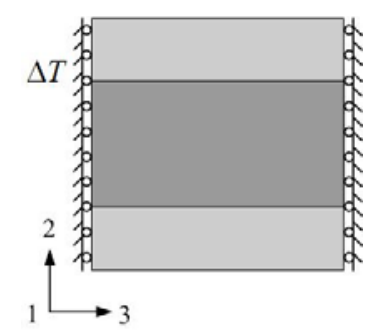

(b) 1-dirction cross-section
Fig. 3. RVC under boundary condition 2

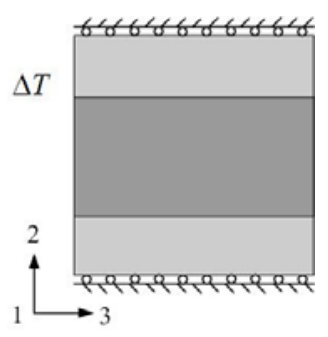

(a) 1-dirction cross-section Fig. 4. RVC under boundary condition 3

The RVC under boundary condition 1 is restricted to move in the 2, 3 directions but free in the 1 direction, which leads to the deformation caused by the temperature increase is free in the 1 direction while restricted in the 2, 3 direction. The following effective stress and strain conditions can be obtained, note that the superscript (1) represents the first boundary condition:

$$
\bar{\sigma}_{11}^{(1)}=0, \bar{\varepsilon}_{22}^{(1)}=0, \bar{\varepsilon}_{33}^{(1)}=0
$$

Inverting equation (6) into equation (4), one obtains the following expressions:

$$
\begin{aligned}
& \bar{\varepsilon}_{11}^{(1)}=\alpha_{11}^{H} \Delta T+\frac{D_{1122}^{H}}{D_{1111}^{H}} \alpha_{22}^{H} \Delta T+\frac{D_{1133}^{H}}{D_{1111}^{H}} \alpha_{33}^{H} \Delta T \\
& \bar{\sigma}_{22}^{(1)}=\left(\frac{D_{1122}^{H} D_{1122}^{H}}{D_{1111}^{H}}-D_{2222}^{H}\right) \alpha_{22}^{H} \Delta T+\left(\frac{D_{1122}^{H} D_{1133}^{H}}{D_{1111}^{H}}-D_{2233}^{H}\right) \alpha_{33}^{H} \Delta T \\
& \bar{\sigma}_{33}^{(1)}=\left(\frac{D_{1133}^{H} D_{1122}^{H}}{D_{1111}^{H}}-D_{2233}^{H}\right) \alpha_{22}^{H} \Delta T+\left(\frac{D_{1133}^{H} D_{1133}^{H}}{D_{1111}^{H}}-D_{3333}^{H}\right) \alpha_{33}^{H} \Delta T
\end{aligned}
$$

The same demonstrations can be made for the other two boundary conditions, and the following expressions can be obtained:

$$
\begin{aligned}
& \bar{\varepsilon}_{22}^{(1)}=\alpha_{22}^{H} \Delta T+\frac{D_{1122}^{H}}{D_{2222}^{H}} \alpha_{11}^{H} \Delta T+\frac{D_{2233}^{H}}{D_{2222}^{H}} \alpha_{33}^{H} \Delta T \\
& \bar{\sigma}_{11}^{(1)}=\left(\frac{D_{1122}^{H} D_{1122}^{H}}{D_{2222}^{H}}-D_{1111}^{H}\right) \alpha_{11}^{H} \Delta T+\left(\frac{D_{1122}^{H} D_{2233}^{H}}{D_{2222}^{H}}-D_{1133}^{H}\right) \alpha_{33}^{H} \Delta T \\
& \bar{\sigma}_{33}^{(1)}=\left(\frac{D_{2233}^{H} D_{1122}^{H}}{D_{2222}^{H}}-D_{1133}^{H}\right) \alpha_{11}^{H} \Delta T+\left(\frac{D_{2233}^{H} D_{2233}^{H}}{D_{2222}^{H}}-D_{3333}^{H}\right) \alpha_{33}^{H} \Delta T \\
& \bar{\varepsilon}_{33}^{(1)}=\alpha_{33}^{H} \Delta T+\frac{D_{1133}^{H}}{D_{3333}^{H}} \alpha_{11}^{H} \Delta T+\frac{D_{2233}^{H}}{D_{3333}^{H}} \alpha_{22}^{H} \Delta T \\
& \bar{\sigma}_{11}^{(1)}=\left(\frac{D_{1133}^{H} D_{1133}^{H}}{D_{3333}^{H}}-D_{1111}^{H}\right) \alpha_{11}^{H} \Delta T+\left(\frac{D_{1133}^{H} D_{2233}^{H}}{D_{3333}^{H}}-D_{1122}^{H}\right) \alpha_{22}^{H} \Delta T \\
& \bar{\sigma}_{22}^{(1)}=\left(\frac{D_{2233}^{H} D_{1133}^{H}}{D_{3333}^{H}}-D_{1122}^{H}\right) \alpha_{11}^{H} \Delta T+\left(\frac{D_{2233}^{H} D_{2233}^{H}}{D_{3333}^{H}}-D_{2222}^{H}\right) \alpha_{22}^{H} \Delta T \\
& C_{11}^{H}
\end{aligned}
$$

Considering the RVC under boundary condition 1 , the relationship between the strain energy of RVC and homogeneous equivalent model is illustrated below. Here, boundary condition 4 is imposed on the RVC, as shown in Fig. 5. The displacement $u_{1}$ and interface force $f$ are equivalent to the displacement and interface force caused by the thermal deformation under boundary condition 1 , which are expressed as:

$$
\begin{aligned}
& u_{1}=\left(\alpha_{11}^{H} \Delta T+\frac{D_{1122}^{H}}{D_{1111}^{H}} \alpha_{22}^{H} \Delta T+\frac{D_{1133}^{H}}{D_{1111}^{H}} \alpha_{33}^{H} \Delta T\right) l \\
& f=\int_{S}\left[\boldsymbol{D}\left(E_{1}\right) \alpha\left(\alpha_{1}\right)-\boldsymbol{D}\left(E_{2}\right) \alpha\left(\alpha_{2}\right)\right] \Delta T d s
\end{aligned}
$$

Obviously, the RVC under boundary condition 4 has the same deformation field as that under boundary condition 1 and thus the strain energies of RVC under both two boundary conditions are equivalent: 


$$
C_{R}^{(1)}=C_{R}^{(4)}
$$

where $C_{R}^{(1)}$ and $C_{R}^{(4)}$ indicate the strain energy of RVC under boundary condition 1 and 4 , respectively.

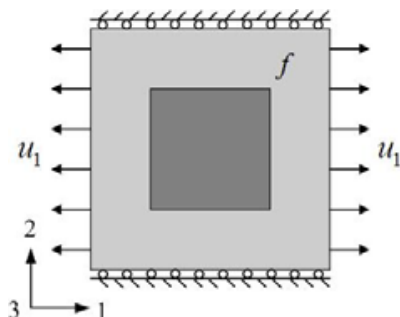

(a) 3-dirction cross-section

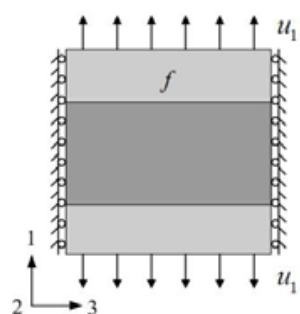

(b) 2-dirction cross-section Fig. 5. RVC under boundary condition 4

Similar demonstration can be made for the homogeneous equivalent model. The equivalent model under boundary condition 1 (depicted in Fig. 6) has the same deformation field as that under boundary condition 5 (depicted in Fig. 7), and the strain energy of equivalent model under these two boundary conditions are equivalent:

$$
C_{H}^{(1)}=C_{H}^{(5)}
$$

where $C_{H}^{(1)}$ and $C_{H}^{(5)}$ refer to the strain energy of homogeneous equivalent model under boundary condition 1 and 5 , respectively.

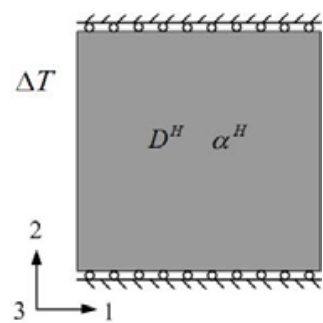

(a) 3-dirction cross-section

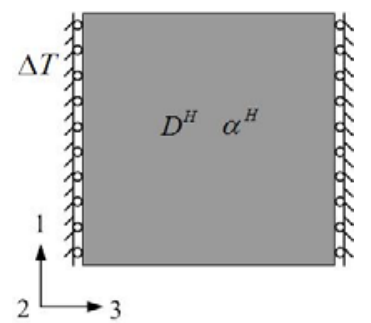

(b) 2-dirction cross-section

Fig. 6. Homogeneous equivalent model under boundary condition 1

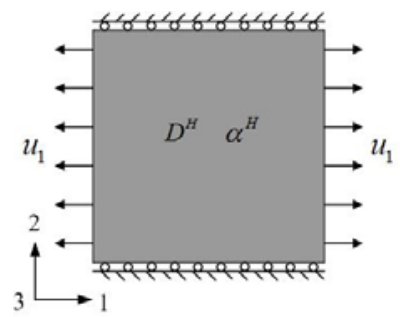

(a) 3-dirction cross-section

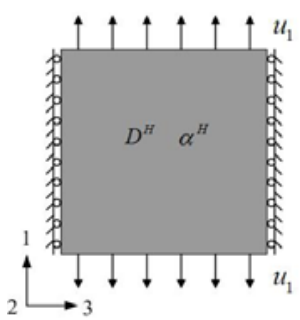

(b) 2-dirction cross-section

Fig. 7. Homogeneous equivalent model under boundary condition 5

It can be demonstrated that the strain energy related to the $\mathrm{RVC}$ is equal to that of the homogeneous equivalent model under uniformly distributed loading boundary conditions. Hence, the strain energy of RVC under boundary condition 5 (depicted in Fig. 8), $C_{R}^{(5)}$, can be expressed as:

$$
C_{R}^{(5)}=C_{H}^{(5)}
$$

Comparing equation (14) with equation (13) results in:

$$
C_{R}^{(5)}=C_{H}^{(1)}
$$
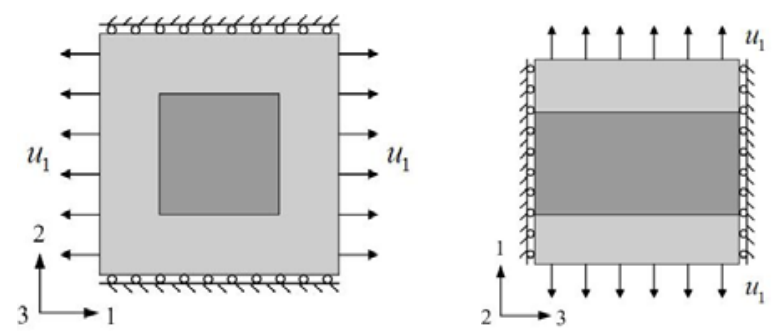

(a) 3-dirction cross-section (b) 2-dirction cross-section Fig. 8. RVC under boundary condition 5

Boundary conditions 6 and 7, as illustrated in Figs 9 and 10 , are then imposed on the RVC respectively. The interface force $f$ denoted in Fig 10 is equivalent to that caused by the thermal deformation under boundary condition 6 . Hence, the strain energies of RVC under both two boundary conditions are equivalent:

$$
C_{R}^{(6)}=C_{R}^{(7)}
$$

where $C_{R}^{(6)}$ and $C_{R}^{(7)}$ refer to the strain energy of RVC under boundary condition 6 and 7 , respectively.

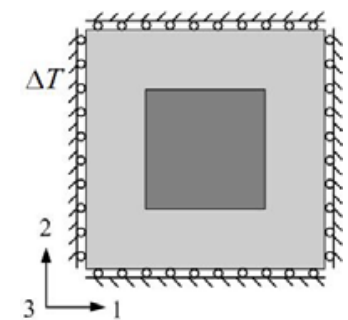

(a) 3-dirction cross-section

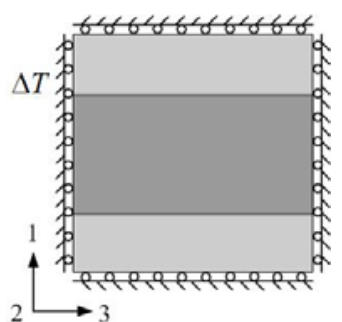

(b) 2-dirction cross-section Fig. 9. RVC under boundary condition 6

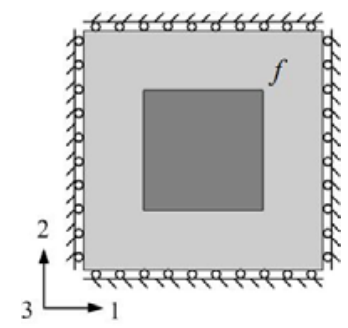

(a) 3-dirction cross-section

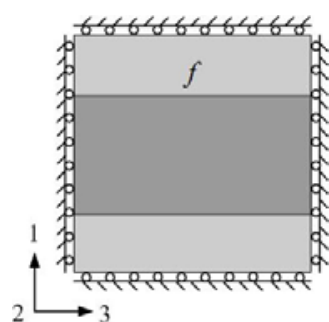

(b) 2-dirction cross-section
Fig. 10. RVC under boundary condition 7

Considering the RVC under boundary conditions 4, 5 and 7, one can explicitly derive the following expression:

$$
C_{R}^{(5)}+C_{R}^{(7)}=C_{R}^{(4)}
$$

Then, the relation between the strain energy of unit cell and homogeneous equivalent model under boundary condition 1 can be obtained by substituting equations (12), (15) and (16) into equation (17):

$$
C_{R}^{(1)}=C_{R}^{(6)}+C_{H}^{(1)}
$$

The same demonstrations can be made to obtain the relationship between the strain energy of unit cell and homogeneous equivalent model under boundary condition 2 :

$$
C_{R}^{(2)}=C_{R}^{(6)}+C_{H}^{(2)}
$$

and boundary condition 3 :

$$
C_{R}^{(3)}=C_{R}^{(6)}+C_{H}^{(3)}
$$

The strain energy of homogeneous equivalent model under boundary conditions 1, 2 and 3 can be easily determined as: 


$$
\begin{aligned}
& C_{H}^{(1)}=D_{1111}^{H}\left(\alpha_{11}^{H} \Delta T+\frac{D_{1122}^{H}}{D_{1111}^{H}} \alpha_{22}^{H} \Delta T+\frac{D_{1133}^{H}}{D_{1111}^{H}} \alpha_{33}^{H} \Delta T\right)^{2} V \\
& C_{H}^{(2)}=D_{2222}^{H}\left(\alpha_{22}^{H} \Delta T+\frac{D_{1122}^{H}}{D_{2222}^{H}} \alpha_{11}^{H} \Delta T+\frac{D_{2233}^{H}}{D_{2222}^{H}} \alpha_{33}^{H} \Delta T\right)^{2} V \\
& C_{H}^{(3)}=D_{3333}^{H}\left(\alpha_{33}^{H} \Delta T+\frac{D_{1133}^{H}}{D_{3333}^{H}} \alpha_{11}^{H} \Delta T+\frac{D_{2233}^{H}}{D_{3333}^{H}} \alpha_{22}^{H} \Delta T\right)^{2} V
\end{aligned}
$$

note that $V$ is the volume of unit cell. By replacing $C_{H}^{(1)}$, $C_{H}^{(2)}$ and $C_{H}^{(3)}$ into equation (18), (19) and (20), respectively, one obtains the following expressions:

$$
\begin{aligned}
& C_{R}^{(1)}-C_{R}^{(6)}=D_{1111}^{H}\left(\alpha_{11}^{H} \Delta T+\frac{D_{1122}^{H}}{D_{1111}^{H}} \alpha_{22}^{H} \Delta T+\frac{D_{1133}^{H}}{D_{1111}^{H}} \alpha_{33}^{H} \Delta T\right)^{2} V \\
& C_{R}^{(2)}-C_{R}^{(6)}=D_{2222}^{H}\left(\alpha_{22}^{H} \Delta T+\frac{D_{1122}^{H}}{D_{2222}^{H}} \alpha_{11}^{H} \Delta T+\frac{D_{2233}^{H}}{D_{2222}^{H}} \alpha_{33}^{H} \Delta T\right)^{2} V \\
& C_{R}^{(3)}-C_{R}^{(6)}=D_{3333}^{H}\left(\alpha_{33}^{H} \Delta T+\frac{D_{1133}^{H}}{D_{3333}^{H}} \alpha_{11}^{H} \Delta T+\frac{D_{2233}^{H}}{D_{3333}^{H}} \alpha_{22}^{H} \Delta T\right)^{2} V
\end{aligned}
$$

Solving equations (24)-(26) finally yields the effective CTES of RVC:

$$
\begin{aligned}
& \left(\frac{D_{1122}^{H}}{D_{1111}^{H}} \frac{D_{2233}^{H}}{D_{2222}^{H}}-\frac{D_{1133}^{H}}{D_{1111}^{H}}\right)\left(\frac{D_{2233}^{H}}{D_{3333}^{H}} Q-R\right)- \\
& \alpha_{11}^{H}=\frac{\left(\frac{D_{2233}^{H}}{D_{3333}^{H}} \frac{D_{2233}^{H}}{D_{2222}^{H}}-1\right)\left(\frac{D_{1122}^{H}}{D_{1111}^{H}} Q-P\right)}{\left(\frac{D_{1122}^{H}}{D_{1111}^{H}} \frac{D_{2233}^{H}}{D_{2222}^{H}}-\frac{D_{1133}^{H}}{D_{1111}^{H}}\right)\left(\frac{D_{2233}^{H}}{D_{3333}^{H}} \frac{D_{1122}^{H}}{D_{2222}^{H}}-\frac{D_{1133}^{H}}{D_{3333}^{H}}\right)-} \\
& \left(\frac{D_{2233}^{H}}{D_{3333}^{H}} \frac{D_{2233}^{H}}{D_{2222}^{H}}-1\right)\left(\frac{D_{1122}^{H}}{D_{1111}^{H}} \frac{D_{1122}^{H}}{D_{2222}^{H}}-1\right) \\
& \left(\frac{D_{1122}^{H}}{D_{2222}^{H}} \frac{D_{1133}^{H}}{D_{1111}^{H}}-\frac{D_{2233}^{H}}{D_{2222}^{H}}\right)\left(\frac{D_{1133}^{H}}{D_{3333}^{H}} P-R\right)- \\
& \alpha_{22}^{H}=\frac{\left(\frac{D_{1133}^{H}}{D_{3333}^{H}} \frac{D_{1133}^{H}}{D_{1111}^{H}}-1\right)\left(\frac{D_{1122}^{H}}{D_{2222}^{H}} P-Q\right)}{\left(\frac{D_{1122}^{H}}{D_{2222}^{H}} \frac{D_{1133}^{H}}{D_{1111}^{H}}-\frac{D_{2233}^{H}}{D_{2222}^{H}}\right)\left(\frac{D_{1133}^{H}}{D_{3333}^{H}} \frac{D_{1122}^{H}}{D_{1111}^{H}}-\frac{D_{2233}^{H}}{D_{3333}^{H}}\right)-} \\
& \left(\frac{D_{1133}^{H}}{D_{3333}^{H}} \frac{D_{1133}^{H}}{D_{1111}^{H}}-1\right)\left(\frac{D_{1122}^{H}}{D_{2222}^{H}} \frac{D_{1122}^{H}}{D_{1111}^{H}}-1\right) \\
& \left(\frac{D_{1133}^{H}}{D_{3333}^{H}} \frac{D_{1122}^{H}}{D_{1111}^{H}}-\frac{D_{2233}^{H}}{D_{3333}^{H}}\right)\left(\frac{D_{1122}^{H}}{D_{2222}^{H}} P-Q\right)- \\
& \alpha_{33}^{H}=\frac{\left(\frac{D_{1122}^{H}}{D_{2222}^{H}} \frac{D_{1122}^{H}}{D_{1111}^{H}}-1\right)\left(\frac{D_{1133}^{H}}{D_{3333}^{H}} P-R\right)}{\left(\frac{D_{1133}^{H}}{D_{3333}^{H}} \frac{D_{1122}^{H}}{D_{1111}^{H}}-\frac{D_{2233}^{H}}{D_{3333}^{H}}\right)\left(\frac{D_{1122}^{H}}{D_{2222}^{H}} \frac{D_{1133}^{H}}{D_{1111}^{H}}-\frac{D_{2233}^{H}}{D_{2222}^{H}}\right)-} \\
& \left(\frac{D_{1122}^{H}}{D_{2222}^{H}} \frac{D_{1122}^{H}}{D_{1111}^{H}}-1\right)\left(\frac{D_{1133}^{H}}{D_{3333}^{H}} \frac{D_{1133}^{H}}{D_{1111}^{H}}-1\right)
\end{aligned}
$$

where $P=\sqrt{\frac{C_{R}^{(1)}-C_{R}^{(6)}}{D_{1111}^{H}(\Delta T)^{2} V}}, \quad Q=\sqrt{\frac{C_{R}^{(2)}-C_{R}^{(6)}}{D_{2222}^{H}(\Delta T)^{2} V}} \quad$ and $R=\sqrt{\frac{C_{R}^{(3)}-C_{R}^{(6)}}{D_{3333}^{H}(\Delta T)^{2} V}} \cdot$ Regarding the above equations, an important point to be noted is that the explicit closed-form expressions can be established between the effective CTEs and the elastic tensors by virtue of the strain energy model. In practice, the considered RVC will be discretized into a finite element model on which the above four boundary conditions 1,2, 3 and 6 will be imposed to evaluate the corresponding strain energies, respectively.

\section{Multi-scale modeling of RVC}

The 3D 4-step braided $\mathrm{C} / \mathrm{SiC}$ composite is considered in the present study. The RVC model of the 3D 4-step braided $\mathrm{C} / \mathrm{SiC}$ composite involves two scales: fiber scale and tow scale. The first scale concerns the modeling of RVC for tows and the second scale concerns the modeling of RVC for $3 \mathrm{D}$ 4-step braided composites. Considering the multi-scale characteristics, the multi-scale analysis procedure are carried out for the analysis: (a). On the fiber scale, finite element model is built to obtain the effective thermo-elastic properties of the fiber-scale RVC, and the results are used for the tows that are treated as a homogeneous transversely isotropic material. (b). Finite element model of the tow-scale RVC is created to evaluate effective thermo-elastic properties of the composite. Here, RVC models of both scales are established using ANSYS finite element software.

\subsection{Fiber-scale model}

The fiber-scale concerns the tows which are considered as unidirectional fiber reinforced composites. Fig. 11 shows the finite element model of the RVC with a $60 \%$ fiber volume fraction of $\mathrm{C} / \mathrm{SiC}$ unidirectional composite. Similar finite element models can be created for other volume fractions.

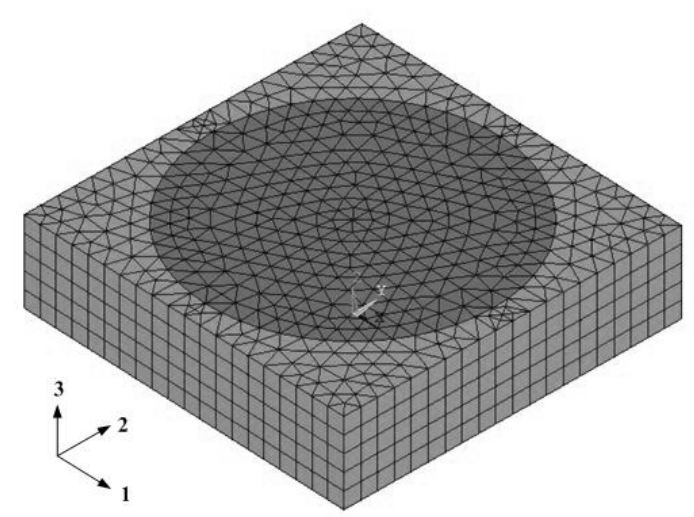

Fig. 11. Finite element model of the fiber-scale RVC

\subsection{Tow-scale model}

Over the last decades, extensive investigations have been mainly carried out [2-12] about the modeling of 3D braided composites. 4-step method is widely used as the basic forming technique of 3D braiding composites. In the 4-step braiding process, the braider tows are intertwined through their relative displacements. The braiding pattern is termed $1 \times 1$ because of the identical distance traveled by all the rows or columns in each step. Four steps of motion constitute one machine cycle which will braid the tows. As these steps of motion are repeated, the tows move throughout the crosssection and are interlaced to form the braided structure. More details about the 4-step braiding process can be found in Ref. [6].

The RVC structure used in this paper is followed from which described in Ref. [12]: the cross-section of tow is hex- 
agonal; the braided structure is uniform and all the tows are straight within the braided preform; all tows in the braided preform have identical constituent material, size and flexibility. Fig. 12 shows the finite element model of the RVC of a $22^{\circ}$ braiding angle 3D 4 -step braided $\mathrm{C} / \mathrm{SiC}$ composite.

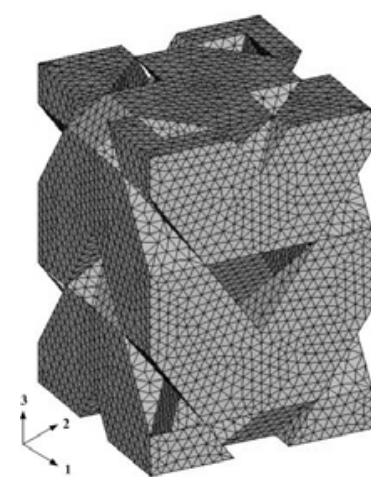

(a) Mesh of the tows

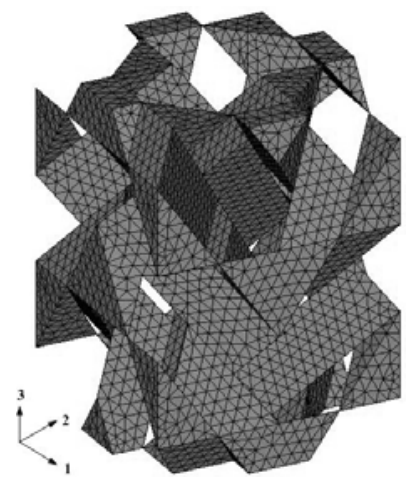

(b) Mesh of the matrix

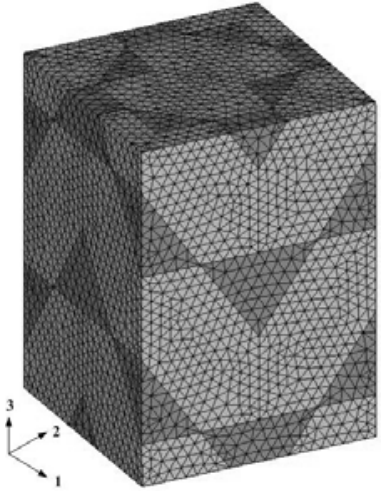

(c) Combined tows and matrix mesh

Fig. 12. Finite element model of the tow-scale RVC

\section{Numerical examples}

The CTE for 3D braided $\mathrm{C} / \mathrm{SiC}$ composite under different temperatures are investigated experimentally [13]. The thermo-elastic properties of its constituents (Carbon fiber, Silicon carbide matrix) under various temperatures are summarized in Table 1.

Table 1. Properties of the constituents

\begin{tabular}{llllll}
\hline & & 200 & 500 & 700 & 800 \\
\hline \multirow{2}{*}{$\mathrm{E}_{11}(\mathrm{GPa})$} & C fiber & 22 & 22 & 22 & 22 \\
& SiC matrix & 350 & 350 & 350 & 350 \\
$\mathrm{E}_{33}(\mathrm{GPa})$ & C fiber & 220 & 220 & 220 & 220 \\
& SiC matrix & 350 & 350 & 350 & 350 \\
$\mathrm{G}_{23}(\mathrm{GPa})$ & C fiber & 4.8 & 4.8 & 4.8 & 4.8 \\
& SiC matrix & 145.8 & 145.8 & 145.8 & 145.8 \\
$v_{12}$ & C fiber & 0.4 & 0.4 & 0.4 & 0.4 \\
& SiC matrix & 0.2 & 0.2 & 0.2 & 0.2 \\
$v_{23}$ & C fiber & 0.12 & 0.12 & 0.12 & 0.12 \\
& SiC matrix & 0.2 & 0.2 & 0.2 & 0.2 \\
$\alpha_{11}\left(10^{-6} /{ }^{\circ} \mathrm{C}\right)$ & C fiber & 8.85 & 8.85 & 8.85 & 8.85 \\
& SiC matrix & 1.9 & 3.7 & 4.6 & 4.9 \\
$\alpha_{33}\left(10^{-6} /{ }^{\circ} \mathrm{C}\right)$ & C fiber & 0.0 & 1.5 & 1.5 & 1.5 \\
& SiC matrix & 1.9 & 3.7 & 4.6 & 4.9 \\
\hline
\end{tabular}

The orthotropic thermo-elastic tow properties are firstly generated using strain energy model, followed by computation of the effective thermo-elastic properties for 3D braided $\mathrm{C} / \mathrm{SiC}$ composite.
In Table2, the CTEs obtained by the strain energy model are compared with experimental results given in Ref. [13]. It can be seen that the predicted results coincide well with the experiments.

Table 2. Comparison of computed CTEs with experimental and numerical results

\begin{tabular}{llcccc}
\hline & & 200 & 500 & 700 & 800 \\
\hline \multirow{2}{*}{$\alpha_{11}\left(10^{-6} /{ }^{\circ} \mathrm{C}\right)$} & $\begin{array}{l}\text { Strain energy } \\
\text { model }\end{array}$ & 3.31 & 4.63 & 5.18 & 5.38 \\
& Experiment & 3.76 & 5.31 & 6.08 & 6.53 \\
\hline \multirow{2}{*}{$\alpha_{33}\left(10^{-6} /{ }^{\circ} \mathrm{C}\right)$} & Strain energy & 1.79 & 3.26 & 3.64 & 3.77 \\
& model & 1.67 & 3.02 & 3.98 & 4.70 \\
\hline
\end{tabular}

\section{Conclusions}

In this paper, the predicting of effective CTEs for 3D braided $\mathrm{C} / \mathrm{SiC}$ composite is presented. Finite element modeling of the $3 \mathrm{D}$ braided $\mathrm{C} / \mathrm{SiC}$ composite is carried out. The strain energy model is presented as an efficient homogenization approach and effective CTE are obtained based on the sequential homogenization from the fiber scale to the tow scale. The significance of strain energy model lies in its simplicity in numerical implementation. In addition, by applying the strain energy model, the closed-form expressions between the effective CTEs and the elastic tensors can be established explicitly. The comparison with the experimental results shows the validity and the rationality of the present model.

\section{References}

1. R. Naslain. Design, preparation and properties of nonoxide CMCs for application in engines and nuclear reactors: an overview. Compos. Sci. Technol. 64, 155-170 (2004).

2. Y.Q. Wang, A.S.D. Wang. On the topological yarn structure of 3-D rectangular and tubular braided performs. Compos. Sci. Technol. 51, 575-586 (1994).

3. Y.Q. Wang, A.S.D. Wang. Microstructure/property relationships in three dimensional braided fiber composites. Compos. Sci. Technol. 53, 213-222 (1995).

4. R. Pandey, H.T. Hahn. Designing with 4-step braided fabric composites. Compos. Sci. Technol. 56, 623-634 (1996).

5. H.Y. Sun, X. Qiao. Prediction of the mechanical properties of three-dimensionally braided composites. Compos. Sci. Technol. 57, 623-629 (1997).

6. L. Chen, X.M. Tao, C.L. Choy. On the microstructure of three dimensional braided preforms. Compos. Sci. Technol. 59, 391-404 (1999).

7. L. Chen, X.M. Tao, C.L. Choy. Mechanical analysis of 3$D$ braided composites by the finite multiphase element method. Compos. Sci. Technol. 59, 2383-2391 (1999).

8. Z.X. Tang, R. Postle. Mechanics of three-dimensional braided structures for composite materials-part : fabric structure and fibre volume fraction. Compos. Struct. 49, 451-459 (2000).

9. Z.X. Tang, R. Postle. Mechanics of three-dimensional braided structures for composite materials-part II: pre- 
diction of the elastic moduli. Compos. Struct. 51, 451-457 (2001).

10. X.K. Sun, C.J. Sun. Mechanical properties of threedimensional braided composites. Compos. Struct. 65, 485-492 (2004).

11. K. Xu, X.W. Xu. Finite element analysis of mechanical properties of $3 D$ five-directional braided composites. Mater. Sci. Eng. A 487: 499-509 (2008).

12. G.D. Fang, J. Liang. B.L. Wang. Progressive damage and nonlinear analysis of $3 D$ four-directional braided composites under unidirectional tension. Compos. Struct. 89, 126-133 (2009).

13. Q. Zhang, L.F. Cheng, L.T. Zhang, et al. Thermal expansion behavior of carbon fiber reinforced chemical-vaporinfiltrated silicon carbide composites from room temperature to $1400{ }^{\circ} \mathrm{C}$. Mater. Lett. 60, 3245-3247 (2006).

14. Y.J. Xu, W.H. Zhang, H.B. Wang. Prediction of effective elastic modulus of plain weave multiphase and multilayer silicon carbide ceramic matrix composite. Mater. Sci. Technol. 24, 435-442 (2008). 